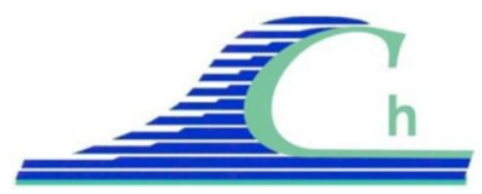

XII ${ }^{\text {èmes }}$ Journées Nationales Génie Côtier - Génie Civil

Cherbourg, 12-14 juin 2012

DOI:10.5150/jngcgc.2012.081-M C Editions Paralia CFL

disponible en ligne - http://www.paralia.fr - available online

\title{
Propagation de la houle en présence d'un mur poreux constitué par plusieurs couches
}

\section{Soumia MORDANE ${ }^{1}$, Ech-Charki DARDAB ${ }^{3}$, Nora EL AMRI ${ }^{2}$, Mohamed CHAGDALI ${ }^{1}$}

1. Faculté des Sciences, Ben M’Sik, Université Hassan II, Casablanca, Maroc. m_chagdali@hotmail.com

3. Ecole Royale Naval, Maroc.

2. FST Settat Maroc.

\section{Résumé :}

Dans ce travail, on s’intéresse à l'étude hydrodynamique de l'écoulement généré par l'interaction de la houle avec un mur vertical perméable constitué de plusieurs couches. Chaque couche est caractérisée par ses propres dimensions géométriques, son coefficient de perméabilité et sa porosité. Ces couches peuvent être disposées d'une manière verticale. La houle considérée est prise dans le cadre de la théorie potentielle. L'approche est analytique et les modèles considérés sont au premier ordre. Les résultats obtenus concernent l'écriture généralisée du coefficient de réflexion. Ce calcul est pertinent pour le dimensionnement des digues verticales et des digues mixtes.

Mots clés :

Houle - Milieu poreux - Digue verticale - Multicouche - Réflexion

\section{Introduction}

Différentes types de structures sont proposées pour l'atténuation de l'effet de la houle à la côte. Dans une structure poreuse, l'atténuation de la houle se fait essentiellement par dissipation de l'énergie. Parmi ces structures, les digues verticales poreuses montrent une efficacité et un pouvoir d'atténuation important et une réduction de l'action de la pression sur l'ouvrage. La théorie de SOLLITT \& CROSS (1972) sert comme base pour modéliser les écoulements dans les milieux poreux. Cette théorie a été également appliquée avec succès à l'analyse des ondes longues par MADSEN (1974). LEE (1987) a développé une méthode généralisée pour des milieux poreux avec des couches multiples. DALRYMPLE et al. (1991) ont prolongé les solutions aux structures soumises à une houle d'incidence oblique.

Dans ce travail, on s’intéresse à l'étude hydrodynamique de l'écoulement généré par l'interaction de la houle avec un mur vertical poreux constitué de plusieurs couches de caractéristiques géométriques et physiques différentes (figure 1). Le but est de trouver le moyen de choisir la meilleure disposition des couches poreuses en vue d'un meilleur fonctionnement dans la houle. 


\section{Formulation théorique}

On considère une houle monochromatique se propageant en présence d'un mur vertical poreux constituée de $\mathrm{n}$ couches de la même largeur $b$. Le domaine d'étude est constitué d'un écoulement externe, mentionné par la notation $(.)_{01}$ et $(.)_{02}$, et d'un milieu poreux constitué de $n$ couches avec des épaisseurs constantes (figure 1). Ces couches sont définies dans le domaine géométrique suivant :

$0<x<b ; \quad-h_{j} \leq z \leq-h_{j-1} ; j=1,2, \ldots . n$ et $h_{0}=0$

Le problème étudié peut être formulé en termes de potentiel des vitesses $\varphi$ sous la forme (SOLLITT \& CROSS, 1972) :

$$
\begin{array}{ll}
\Delta \varphi_{j}=0 & \text { Dans le domaine de l'écoulement } \\
\frac{\partial \varphi_{j}}{\partial z}=0 & \text { Sur le fond } z=-h \\
\frac{\partial \varphi_{j}}{\partial z}-\frac{\omega^{2}}{g}\left(s_{j}-i f_{j}\right) \varphi_{j}=0 & \text { Sur la surface libre } z=0
\end{array}
$$

où $i$ est l'unité imaginaire $\left(i^{2}=-1\right)$ le nombre complexe, $s_{j}$ et $f_{j}$ sont respectivement le coefficient d'inertie et le coefficient de dissipation de la couche $j$, $g$ est l'accélération de la pesanteur et $\omega$ est la pulsation de l'onde incidente.

Pour le milieu externe le coefficient d'inertie $s_{j}$ est égal à 1 et le coefficient de dissipation $f_{j}$ est égal à 0 .

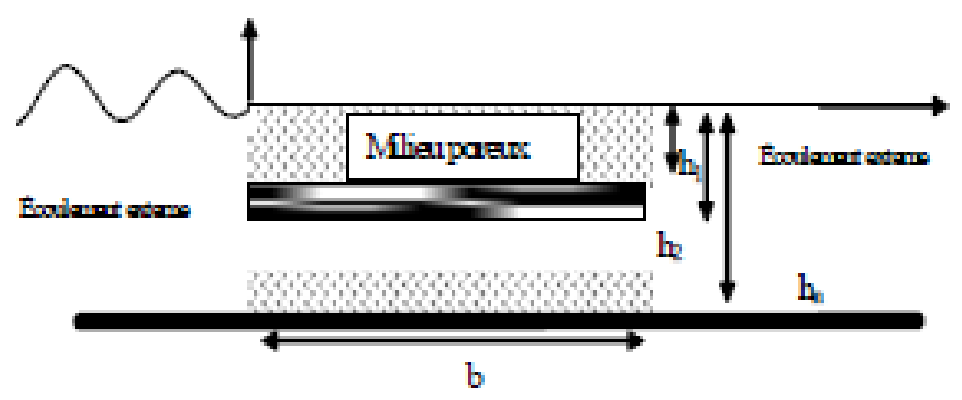

Figure 1. Schéma descriptif du domaine d'étude.

\section{Résolution analytique}

La procédure analytique est composée de trois étapes :

Etape 1 : On subdivise le domaine d'étude en plusieurs sous domaines. Ainsi on aura les deux sous domaines du milieu externe $D_{01}$ et $D_{02}$ qui contiennent respectivement les ondes incidentes - réfléchies et les ondes transmises. Les autres sous domaines $\left(D_{j} ; j=1,2,3, \ldots n\right)$ sont définis par les différentes couches de l'ouvrage. Ensuite dans chaque sous domaine on écrit la forme générale du potentiel des vitesses (on se restreint aux ondes planes).

$$
\begin{aligned}
& \text { Domaine externe }-\infty<x<0 ; \quad-h_{0} \leq z \leq 0 \\
& \varphi_{01}(x, z)=I_{0}(z)\left(e^{-i k x}+R e^{i k x}\right)
\end{aligned}
$$




\section{XII èmes Journées Nationales Génie Côtier - Génie Civil}

Cherbourg, 12-14 juin 2012

Domaine externe $0<x<+\infty ; \quad-h_{0} \leq z \leq 0$

$\varphi_{02}(x, z)=I_{0}(z) T e^{-i k(x-b)}$

Milieu poreux $0<x<b ;-h_{j} \leq z \leq-h_{j-1}$

$\varphi_{j}(x, z)=F_{j}(z)\left(A_{j} e^{-i K x}+B_{j} e^{+i K(x-b)}\right)$

où $R$ et $T$ sont les coefficients de réflexion et de transmission. $A_{j}$, $B_{j}$, et $T$ sont des constantes à déterminer, $k$ et $K$ sont les nombres d'ondes respectivement dans le milieu externe et dans le milieu poreux.

Etape 2 : Dans la seconde étape, on écrit les conditions de raccordement entre les sous domaines :

Continuité de la pression.

$\varphi_{0 I}(0, z)=\left(S_{i}-i f_{i}\right) \varphi_{i}(0, z)$

$\left(S_{j}-i f_{j}\right) \varphi_{j}(b, z)=\varphi_{02}(b, z)$

Continuité de la vitesse.

$\frac{\partial \varphi_{01}(0, z)}{\partial x}=\varepsilon_{j} \frac{\partial \varphi_{j}(0, z)}{\partial x}$
$\frac{\partial \varphi_{02}(b, z)}{\partial x}=\varepsilon_{j} \frac{\partial \varphi_{j}(b, z)}{\partial x}$

Etape 3: On résout un système d'équations algébriques. La résolution permet d'obtenir les expressions du coefficient de réflexion $R$ et de transmission $T$ :

$$
\begin{aligned}
& R=\frac{i\left(1-m_{n}^{2}\right) \sin (K b)}{2 m_{n} \cos (K b)+i\left(1+m_{n}^{2}\right) \sin (K b)} \\
& T=\frac{2 m_{n}}{2 m_{n} \cos (K b)+i\left(1+m_{n}^{2}\right) \sin (K b)}
\end{aligned}
$$

avec :

$$
m_{n}=\frac{K}{k} \frac{1}{Z_{n}} \frac{\Gamma_{n}}{X_{n}} \frac{\sum_{j=1}^{n} \varepsilon_{j} X_{j}}{\sum_{j=1}^{n} \Gamma_{i}} ; Z_{n}=s_{n}-i f_{n} ; \Gamma_{n}=\int_{-h_{n}}^{-h_{n-1}} I_{0}^{2}(z) d z ; X_{j}=\int_{-h_{j}}^{-h_{j-1}} I_{0}(z) F_{j}(z) d z
$$

Les expressions des coefficients $F_{j}(z), I_{0}(z)$ et $m_{n}$ seront explicitées dans le cas monocouche, le cas bicouche et le cas tri couche.

\section{Résultats}

\subsection{Cas d'une seule couche}

Dans ce cas les coefficients $I_{0}(z)$ et $F_{j}(z)$ sont : $I_{0}(z)=\frac{i \omega}{k} \frac{\cosh \left(k\left(z+h_{1}\right)\right)}{\sinh \left(k h_{1}\right)}$ et $F_{1}(z)=\frac{i \omega a}{K} \frac{\cosh \left(K\left(z+h_{1}\right)\right)}{\sinh \left(K h_{1}\right)}$ 
$k$ et $K$ vérifient respectivement les relations de dispersion suivantes :

$\omega^{2}=\operatorname{gktanh}\left(k h_{1}\right)$

$\omega^{2}\left(s_{1}-i f_{1}\right)=g \operatorname{Ktanh}\left(K h_{1}\right)$

Le coefficient de réflexion est :

$R=\frac{i\left(1-m_{1}^{2}\right) \sin (K b)}{2 m_{1} \cos (K b)+i\left(1+m_{1}^{2}\right) \sin (K b)}$

Le coefficient de transmission :

$T=\frac{2 m_{1}}{2 m_{1} \cos (K b)+i\left(1+m_{1}^{2}\right) \sin (K b)}$

avec :

$m_{1}=\frac{\varepsilon_{1}}{Z_{1}} \frac{K}{k}$

Sur la figure 2 on représente le coefficient de réflexion en fonction de $k h$ pour différentes largeurs du mur poreux.

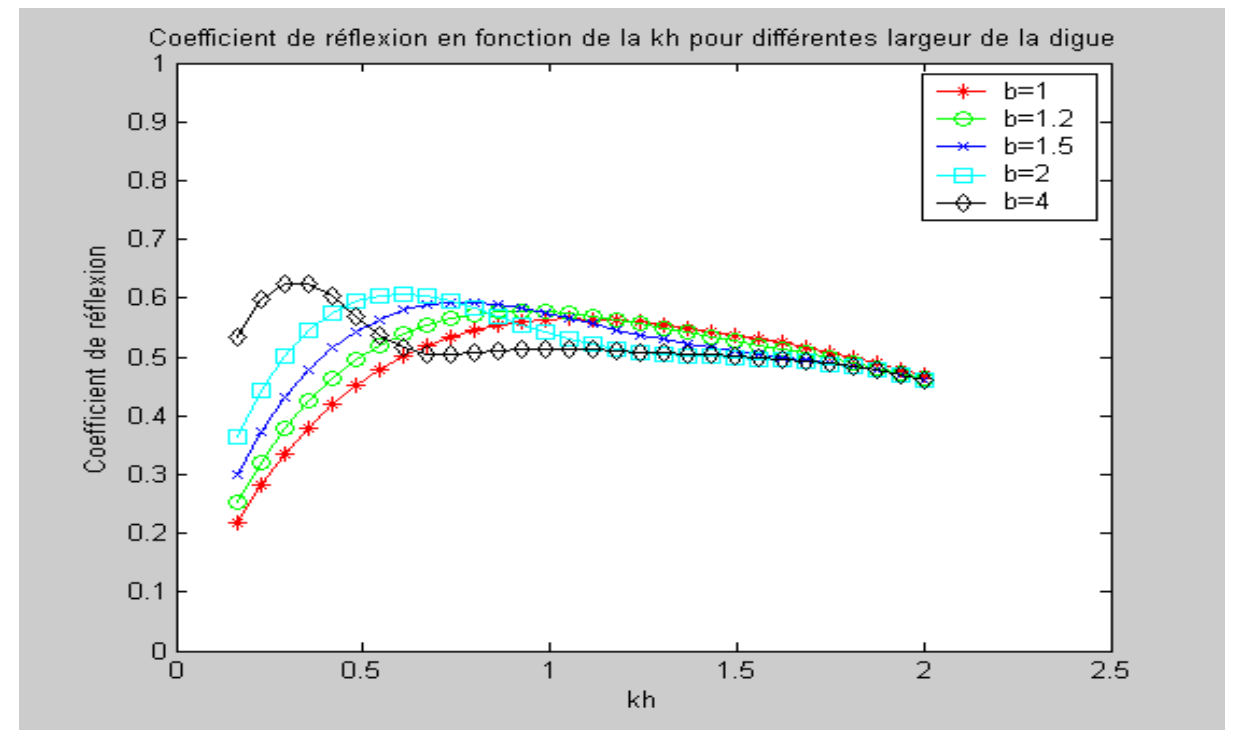

Figure 2. Coefficient de réflexion en fonction de kh pour différentes largeurs de la digue avec $\varepsilon=0.4$.

Lorsqu'on augmente la largeur du mur le maximum du coefficient de réflexion augmente mais la bande de réflexion devient plus étroite. En annexe (section 7, figure 5) les mêmes résultats sont présentés en fonction du rapport largeur - longueur d'onde. Le fonctionnement du mur est conditionné par la dissipation du milieu poreux comme cela est aussi illustré en annexe (section 7, figure 6) par l'évolution du coefficient de transmission dans les mêmes conditions 


\section{XII ${ }^{\text {èmes }}$ Journées Nationales Génie Côtier - Génie Civil \\ Cherbourg, 12-14 juin 2012}

4.2 Cas d'un mur à deux couches

$\omega^{2}=\frac{g K}{Z_{1}} \frac{\tanh \left(K h_{1}\right)+\frac{\varepsilon_{2}}{\varepsilon_{1}} \frac{Z_{1}}{Z_{2}} \tanh K\left(h_{2}-h_{1}\right)}{1+\frac{\varepsilon_{2}}{\varepsilon_{1}} \frac{Z_{1}}{Z_{2}} \tanh \left(K h_{1}\right) \tanh K\left(h_{2}-h_{1}\right)}$

Les résultats présentés sur la figure 3 montrent l'évolution du coefficient de réflexion en fonction de $k h$. La porosité des deux couches étant fixées $\left(\varepsilon_{1}=0.4, \varepsilon_{2}=0.2\right)$. La courbe montre que le coefficient de réflexion de la digue augmente quand l'épaisseur de la couche supérieure diminue pour les valeurs de $k h<1$ et l'inverse se produit pour $k h>1$. Pour la valeur de $k h=1$, on a explicitement la relation $h \approx \lambda / 6$ qui correspond à la limite de l'interaction de la houle avec le fond. L'effet de la seconde couche est important, en particulier pour $h_{1}=0,8$.

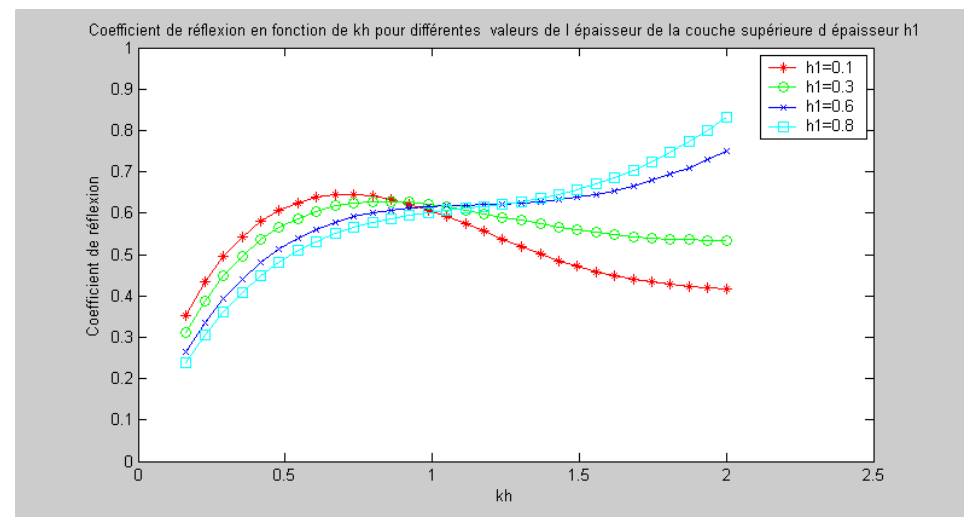

Figure 3. Coefficient de réflexion en fonction de kh pour différentes valeurs de l'épaisseur de la couche supérieure d'épaisseur $h_{1}, \varepsilon_{1}=0.4, \varepsilon_{2}=0.2$.

4.3 Cas d'un milieu à trois couches

Sur la figure 4, on présente le coefficient de réflexion en fonction de $k h$.

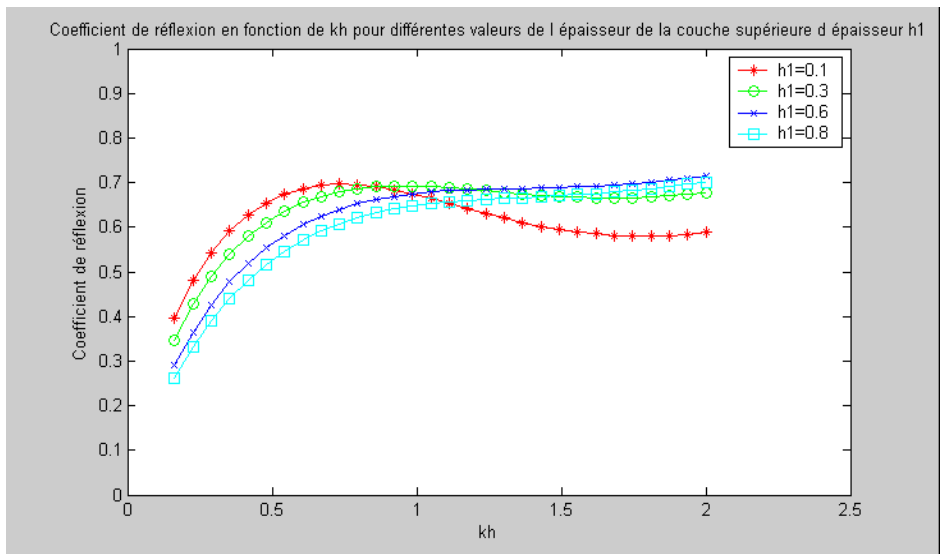

Figure 4. Coefficient de réflexion en fonction de kh pour différentes valeurs de l'épaisseur de la couche supérieure $h_{1} \cdot\left(\varepsilon_{1}=0.4, \varepsilon_{2}=0.2, \varepsilon_{3}=0.1, h_{2}=2 / 3, h_{3}=1\right)$. 


\section{Approximation des ondes longues}

En développant la même méthodologie de calcul pour le cas où la digue est constituée de $\mathrm{n}$ couches de caractéristiques géométriques différentes et en faisant l'approximation d'onde longue ( $K h$ petit) on peut généraliser la relation de dispersion à un nombre fini de couches :

$\varepsilon_{1} \frac{\omega^{2}}{g K}=\sum_{j=1}^{n} \frac{\varepsilon_{j}}{Z_{j}} K\left(h_{j}-h_{j-1}\right)$

Dans ce cas particulier, les coefficients de réflexion et de transmission se généralisent aussi sous la forme :

$R=\frac{i \Lambda}{1+i \Lambda} ; T=\frac{1}{1+i \Lambda}$

avec :

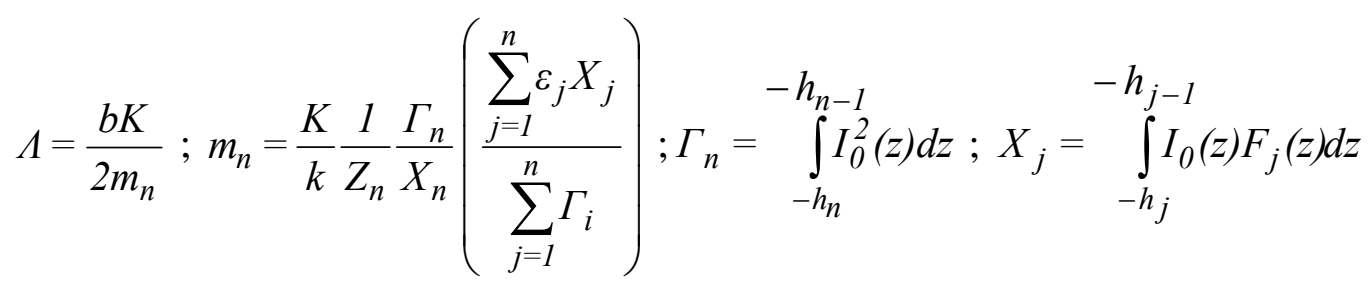

\section{Conclusion}

Dans ce travail, nous nous sommes intéressés à la réflexion de la houle par une digue verticale poreuse constituée de plusieurs couches disposées d'une manière verticale. L'étude analytique a été menée dans le cadre de la théorie potentielle de la houle. Dans le cas de la digue poreuse bicouche et tri couche disposées verticalement, les résultats ont montré d'une part que le coefficient de réflexion augmente quand la porosité des deux milieux diminue pour des épaisseurs des couches fixées. D’autre part, le coefficient de réflexion augmente quand l'épaisseur de la couche supérieure diminue pour des porosités fixes.

Dans le cas de la théorie des ondes longues, nous avons proposé des relations généralisant l'expression des coefficients de réflexion et de transmission pour des milieux constitués de plusieurs couches horizontales. Une étude paramétrique est nécessaire afin d'exploiter les résultats obtenus. Une comparaison avec des résultats d'expérience pourrait apporter des améliorations à la démarche entreprise.

\section{Annexe : Coefficient de réflexion en fonction du rapport largeur sur longueur d'onde.}

Sur la figure 5, on représente le coefficient de réflexion en fonction de $K b$ ( $K$ est le nombre d'onde et $b$ la largeur du mur). 


\section{XII ${ }^{\text {èmes }}$ Journées Nationales Génie Côtier - Génie Civil \\ Cherbourg, 12-14 juin 2012}

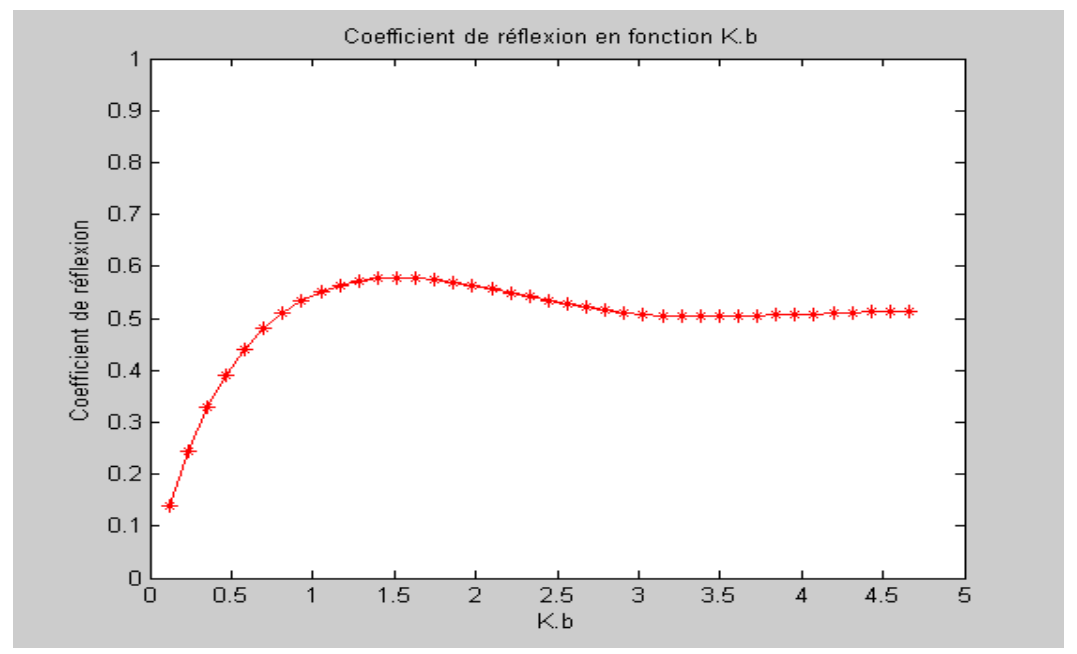

Figure 5. Coefficient de réflexion en fonction $K b$.

Lorsque la largeur de la digue augmente, le coefficient de réflexion augmente vers un maximum, et à partir de $K b=3$ les valeurs restent inchangées.

Sur la figure 6 , on représente respectivement les coefficients de réflexion $R$ et de transmission $T$ en fonction de $k h$ pour une porosité égale à 0.4 .

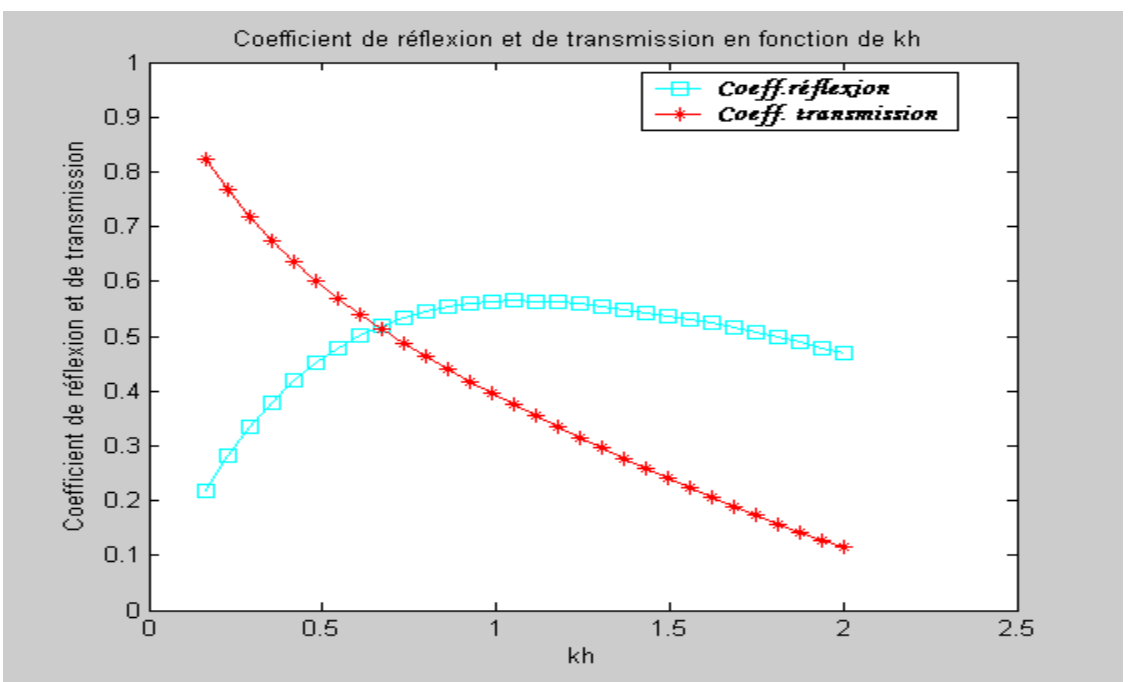

Figure 6. Coefficient de réflexion en fonction de kh pour une valeur de la porosité=0.4.

\section{References}

DALRYMPLE R.A., LOSADA M.A., MARTIN P.A. (1991). Reflection and transmission from porous structures under oblique wave attack. J. Fluid Mech. 224, pp 625-644. doi:10.1017/S0022112091001908

MADSEN O.S. (1974). Wave transmission through porous structures. J. WaterWays, Port, Coastal, Ocean Engng Div., ASCE 100, pp 169-188. 
Thème 4-Ouvrages portuaires et offshore

SOLLITT C.K., CROSS R.H. (1972). Wave transmission through permeable breakwaters. In Proceedings of the 13th International Conference on Coastal Engineering, III, pp 1827-1846.

LEE C.P. (1987). Wave interaction with permeable structures, Ph.D. Thesis, Oregon State University, Corvallis, OR, USA. 